\title{
Was ist ein Notfall?
}

Markus Gassner-Bachmann

Korrespondenz:

Dr. med. Markus Gassner-Bachmann Facharzt für Innere Medizin FMH speziell Allergologie und klinische Immunologie Spitalstrasse 8

CH-9472 Grabs

m.gassner@hin.ch

\section{Einleitung}

Nicht erst seit der Einführung des TARMED bestehen versicherungspolitische Wünsche nach der Definition eines Notfalles. Heute werden Notfallstationen mit Steuergeldern aufgebaut, andererseits ist der Notfalldienst auf dem Lande vielerorts wegen der Überalterung praktizierender Ärzte vor dem Zusammenbrechen.

Not bedeutet einen Zustand der äusseren oder inneren Bedrängnis, der Hilflosigkeit (Brockhaus). Der Notfall ist das Ereignis, das die subjektiv erlebte Bedrängnis oder Hilflosigkeit produziert und rasche «not-wendige» Massnahmen erfordert.

Bei einem medizinischen Notfall handelt es sich um einen Patienten, der plötzlich erkrankt oder eine Krankheit unmittelbar registriert, die eine rasche medizinische Beurteilung oder Behandlung notwendig macht. Daraus ergibt sich, dass dies sicher nicht nur Menschen betrifft, die rasch gerettet werden müssen (z. B. schwerer Unfall, Kind mit akuter Meningitis), sondern auch solche, die mehr oder weniger dringlich ohne Rettungswesen vom Notfallarzt behandelt oder weitergewiesen werden (Fremdkörper im Auge, Hörsturz infolge Cerumen, Läuse usw.).

Weder der Arzt noch der Patient soll à priori einen Notfall definieren. Die heute im ärztlichen Notfalldienst häufige Frage am Telefon, oft vor einer Namensnennung: «Sie haben Notfalldienst - oder?» belegt, dass auch das Bedürfnis des Patienten sich nicht generell als Notfallkriterium eignet. Der Notfalldienst wird oft missbraucht, indem damit bequem auf Kosten anderer oder der Sozialversicherung eine nicht notwendige schnelle Behandlung oder ein Transport mitunter sogar klar bewusst verlangt wird. Deshalb sind auch staatliche Institutionen, Funktionäre, Vertreter von Krankenkassen, Vertrauensärzte von Versicherungen, Präventivmediziner, Politiker und am wenigsten Ökonomen nicht in der Lage, als Notfall einzelne Krankheiten oder Situationen prinzipiell zu definieren.

\section{Fehlende Studien}

Analysen der Behandlungen im hausärztlichen Notfalldienst wären überfällig. Untersuchungen aus dem Rettungswesen, wie eine vorgeschlagene Konzeption eines nationalen Forschungsprojektes für präklinische Medizin, sind zwar interessant, decken aber nur einen kleinen Teil des hausärztlichen Notfalles ab.

\section{Qu'est-ce qu'une urgence?}

L'urgence, chez l'être humain, se montre d'une grande diversité. II est quasiment impossible de fixer des critères généraux définissant l'urgence et les mesures prises. Dans une urgence médicale, un petit détail comme un corps étranger dans I'œil, ou alors une attaque cérébrale, remplissent tous deux ces critères. Même la mort demeure la plupart du temps un cas d'urgence, lorsque la détresse individuelle s'arrête et est transmise aux proches.

Le service d'urgence, exécuté par les médecins depuis des millénaires et dans tous les pays, comprend tous ces aspects. Certes, la manière d'aider gagne sans cesse en complexité, mais elle reste d'autant plus liée aux circonstances concomitantes tant géographiques que temporelles.

Die Behandlungen eines Notfalles unterscheiden sich prinzipiell nicht von allen andern ärztlichen Behandlungen (Tab. 1). Die Diagnose und die Therapie müssen nur im Hinblick auf den Zeitfaktor modifiziert werden. Dabei ist das Erfassen von Langzeitrisiken primär nicht notwendig. Die ersten vier Kriterien bilden die Qualität der klassischen Medizin, die mit dem Kriterium 5 gemessen wird, die Evidence-based Medicine. Die drei letzten beschreiben die aktuellen Bedingungen, unter denen dies erfolgte. Leider sind alle diese Kriterien schwierig messbar, vernetzt, wohl deshalb kaum untersucht.

Die Kommission für praxisorientierte Forschung der Schweizerischen Akademie der medizinischen Wissenschaften (RRMA) hatte 2002 eine Untersuchung lanciert unter dem Projekt «Ein Tag Notfalldienst». Es entstanden über 40 vorwiegend narrative Berichte von Ärzten im Notfalldienst. Das ursprüngliche Projekt wurde aus Kostengründen nicht weiterverfolgt.

\section{Beispiele}

Die folgenden Beispiele sind eine Realität der letzten Jahre. Sie sind zwar im einzelnen nicht alltäglich, belegen aber vielgestaltige Aspekte eines hausärztlichen Notfalldienstes. 


\section{Der unbekannte Notfall vor der Haustüre}

An einem Sonntagmorgen kollidierten zwei Schüler auf einem Motorrad mit einem Auto. Sie lagen offensichtlich verletzt 50 Meter (!) von der Haustüre des Notfallarztes auf dem Asphalt. Viele Leute machten erste Hilfe, aber niemand kam auf die Idee, den Notfallarzt beizuziehen. Der Notfallarzt wurde erst auf diesen Unfall vor seiner Haustüre aufmerksam durch den Helikopter, der über sein Haus hinweg in der benachbarten Wiese landete. Die beiden Schüler wurden in ein Zentrumsspital gebracht. Sie lagen logischerweise mindestens eine Viertelstunde medizinisch unbetreut auf dem Asphalt, und alle haben zugeschaut.

\section{Die unkomplizierte Rettung}

Ein Mann läutet in meiner Praxis. Er hat 100 Meter von der Praxis entfernt einen Knaben bewusstlos am Strassengraben liegen gesehen. Ich habe ihn in mein Auto geladen und ins nahe Spital gebracht ohne Formalitäten - stationäre Überwachung wegen Commotio. Keine Folgeschäden. Früher hätte ich den Patienten in der Praxis, später durch die Eltern überwachen lassen. Heute ist dies versicherungspolitisch (Haftpflicht) zu gefährlich geworden.

\section{Der Notfall «Läuse»}

Nach den Ferien, an einem Sonntagabend, findet eine Mutter bei ihrer Tochter im Schulalter Läuse. Ich habe der Mutter ein wirksames Lausmittel abgegeben. Für die Mitschüler wichtig, dass am folgenden Tag keine lebenden Läuse eingeschleppt wurden! Versicherungspolitisch eine teure Bagatelle, vom sozial- und präventivmedizinischen Standpunkt aus gesehen aber durchaus ein vernünftiger Notfall mit einer sehr sinn-

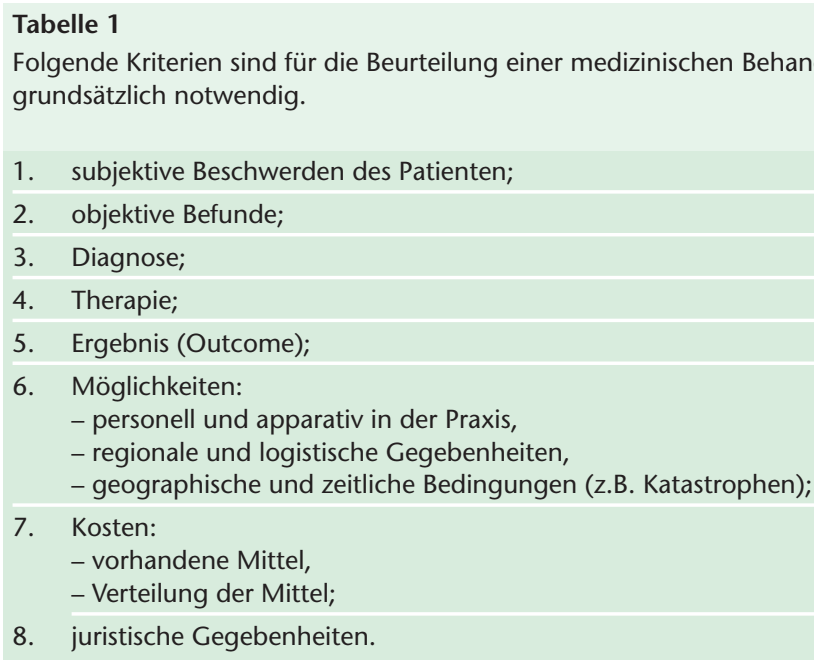

vollen, zeitgerechten, adäquaten und effektiven, also klar not-wendigen Behandlung.

\section{Der Schlaganfall}

Früher war diese Diagnose eine der frustrierendsten, sowohl für den Patienten wie für alle Ärzte. Heute gehört der Schlaganfall zu einem der dringlichsten Notfälle, sofern regional eine Abklärungs- und Therapiemöglichkeit vorhanden ist! Die Therapie und Lebenschance ist von der Geographie abhängig geworden, also ein Notfall nur dort, wo not-wendige Massnahmen zur Verfügung stehen!

\section{Der Todesfall}

Jeder Mensch weiss, dass er irgendwann einmal im Leben diesen Notfall persönlich bewirkt. Irgendein Arzt muss festlegen, wann genau dieser «letzte Notfall» eingetreten ist und weshalb. Als Notfallarzt musste ich gelegentlich notfallmässig ausrücken, wenn der Notarzt (Arzt der Notfallstation, Rega, Ambulanz) oder das entsprechende Team den Tod eines Menschen und die Erfolglosigkeit jeglicher medizinischer Massnahmen zwar festgestellt hat, aber den Tod mit den erwähnten formalistischen Konsequenzen nicht regeln wollte oder konnte. Die Entscheidung, ob allenfalls eine Fremdeinwirkung möglich war, wird dann dem Praktiker auf dem Lande überlassen. Die Todesursache ist oft unsicher. Wenn wir aber diese Zweifel jedes Mal juristisch abklären wollten, würde das Sterben nicht nur teurer, sondern insbesondere unheimlicher und unwürdiger. Aus menschlichen Gründen hütet man sich deshalb manchmal auch, Details zu erfragen oder mehr wissen zu wollen als unbedingt not-wendig.

\section{Vernetzte Zusammenhänge}

Für den Arzt ist jeder Patient ein Notfall, der innert Stunden eine Beratung oder Behandlung benötigt, speziell ausserhalb der üblichen Sprechstundenzeiten.

Massgebend auch im Notfalldienst sind nur, aber alle eingangs erwähnten Kriterien einer ärztlichen Behandlung. So bleiben Kriterien der Patientenzufriedenheit auch heute im Zuge der Marktwirtschaft kaum relevante Details. Als Ärzte behandeln wir doch immer noch Patienten und nicht Kunden. Wie lange wir dies allerdings noch dürfen, wird politisch zunehmend unsicher.

Theoretisch gäbe es für einen Arzt kaum eine abwechslungsreichere Arbeit als den Notfalldienst auf dem Lande. Trotzdem kenne ich persönlich keinen Kollegen, der dies gerne tut. Jeder Notfalldienst ist mit vielen Unsicherheiten be- 
haftet, welche belasten. Frustrationen seitens der Patienten: Wer ist zuständig? Selbsteinweisungen direkt oder indirekt über Notfallnummern, «gute» Ratschläge von Angehörigen, oft gemäss Informationen aus Massenmedien, über Notrufnummern von Krankenkassen; dann beim Notfallarzt: die beschränkten apparativen und personellen Mittel. Am meisten betroffen machen Haftpflicht und Inkassoforderungen wegen fehlender oder ungenügender Hilfeleistung im Notfall. Die heutigen Ansprüche nach Sicherheit verstärken zunehmend dieses Unbehagen.

Moderne Organisationen eines Notarztdienstes bewirken zusätzliche Unsicherheiten. Der Patient möchte sich seinen Arzt auswählen. Die aktuellen politischen Vorstellungen erachten marktwirtschaftliche Kriterien als optimalste Voraussetzungen eines guten Gesundheitswesens. Doch ausgerechnet diese Kriterien sind im Notfalldienst aufgehoben.

Die medizinische Hilfe kostet, speziell im Notfall. Die Unterschiede: Wie, wann, wo, von wem ein Patient mit einem gleichen Notfall, mit gleichem Ergebnis behandelt wird und für wie viel Geld, wann und von wem bezahlt, sind in der Schweiz zweifellos massiv, aber völlig unbekannt.

Das moderne Management des Rettungswesens weckt in der Bevölkerung auch Illusionen, deren Nebenwirkungen nicht beachtet werden wollen. Impfverweigerung: die Masernenzephalitis oder Pneumonie usw. Das Risiko eines Notfalles steigt z.B. durch risikoreiche Sportarten («no risk - no fun»), Missachtung von Verkehrsregeln. Wir bezahlen ja Versicherungsprämien, auch für die Spitzenmedizin! Unfälle werden im Notfall geheilt!?

Ein hausärztlicher Notfalldienst ist dabei nicht einmal mehr notwendig: Der Notfall vor der Haustüre des Notfallarztes ist kein Einzelfall. Andererseits ist es auch evident, dass nicht jegliche heute praktizierte Hilfeleistung auch notwendig ist, z.B. «Rettung» eines Patienten mit einer Daumenverletzung von der Skipiste mit einem Helikopter. Weshalb werden Spitzensportler anders behandelt (z.B. Klavikulafraktur bei Radfahrern)?

Den Notfallarzt beschäftigen auch wirtschaftliche Aspekte. Lohnt sich eine Anschaffung von Reanimationsutensilien, wie beispielsweise Defibrillator, Sauerstoffflaschen usw.? Andererseits ist nicht bekannt, wie häufig solche Apparaturen wirklich notwendig und lebensrettend sind. Wer bezahlt all das? Wenn das Telefon Nr. 144 ohnehin die Rega alarmiert bei einem Unfall neben der Praxis des Notfallarztes. Lohnt sich da noch eine zeitraubende Fortbildung? Deshalb sind generelle strukturelle und organisatorische Empfehlungen insbesondere von Notfallzentren für die
Qualitätssicherung in der Praxis oft unbrauchbar. Der Notfallarzt und Notfallzentren behandeln Patienten mit gleichen notfallmässigen Erkrankungen und Unfällen, aber mit unterschiedlichen personellen und logistischen Mitteln. Den gleichen materiellen und personellen Aufwand für alle zu fordern, wäre unbezahlbar.

Der Notfallarzt in der Praxis ist meist viel beweglicher, kann Hausbesuche machen, mit einfachen Mittel behandeln, Medikamente in der Praxis abgeben. Der Zeitverlust zwischen Notruf und durchgeführter Therapie ist kürzer als in einer Poliklinik. Dies gilt insbesondere für Situationen auf dem Land. Erschwerend und belastend ist, dass der Notfallarzt seine Entscheidungen innert Minuten allein fällen und erst noch allein handeln muss (ohne MPA!). Er kann dabei weder mit Kollegen diskutieren, noch im Internet nachschauen.

In der Schweiz wird die interdisziplinäre Teamarbeit auf einer Notfallstation hochgejubelt. Aber wer hat Zugang zu dieser Medizin, und wie wirkt sie sich aus auf die gesamte Infrastruktur, Ausbildung, Kosten usw.? Amerikanische «emergency rooms» werden gefordert. Wie hat das wohl beim Orkan Katharina in New Orleans funktioniert? Welche dieser Kriterien sind für eine optimale Notfallbehandlung in Ländern der 3. Welt massgebend?

Die Qualität der doch so banalsten ärztlichen Hilfe, ausgerechnet im Notfall, bleibt somit nach wie vor undefinierbar und auch juristisch nicht beurteilbar. Sie ist abhängig von unterschiedlichsten geographischen, soziologischen und zeitlichen Begleitumständen.

\section{Literatur}

- Stalder H. Un jour de garde. Schweiz Ärztezeitung. 2002;83(10):459.

- Gassner M. Ein Tag Notfalldienst im Sommer 2001. Schweiz Ärztezeitung. 2002;83(10):490-1.

- von Planta M. Konzeption eines nationalen Forschungsprojektes für präklinische Medizin. Schweiz Ärztezeitung. 2004;85(13):676-8.

- Arbeitsgruppe der Interessengemeinschaft ärztliche Leiter von Notfallstationen. Strukturelle und organisatorische Empfehlungen für die Qualitätssicherung. Schweiz Ärztezeitung. 2005;86(33):1915-28.

- Medicus Mundi. Health in emergency. Gesundheit in Krise und Konflikt. Bulletin 98; Oktober 2005.

- Matter H, Caduff B, Schöb O. Interdisziplinäre Teamarbeit auf einer Notfallstation. Schweiz Ärztezeitung. 2006;87(19):849-53.

- Stäubli M, Briner V, Hangartner PJ. Modell mit Nachteilen für Patientenbetreuung und Weiterbildung der Assistenzärzte. Schweiz Ärztezeitung. 2006;87(35):1502-4.

- Gassner M. Wollen Kranke wirklich Kunden sein? Schweiz Med Forum. 2006;6:757. 